\title{
Domain of the double sequential band matrix in the classical sequence spaces
}

Murat Candan ${ }^{*}$

\section{"Correspondence:}

murat.candan@inonu.edu.tr;

murat_cndn@hotmail.com

Department of Mathematics,

Faculty of Arts Sciences, İnönü

University, Malatya, 44280, Turkey

\begin{abstract}
Let $\lambda$ denote any one of the classical spaces $\ell_{\infty}, c_{1} c_{0}$ and $\ell_{p}$ of bounded, convergent, null and absolutely $p$-summable sequences, respectively, and $\tilde{\lambda}$ also be the domain of the double sequential band matrix $B(\widetilde{r}, \widetilde{s})$ in the sequence space $\lambda$, where $\left(r_{n}\right)_{n=0}^{\infty}$ and $\left(s_{n}\right)_{n=0}^{\infty}$ are given convergent sequences of positive real numbers and $1 \leq p<\infty$. The present paper is devoted to studying the sequence space $\tilde{\lambda}$. Furthermore, the $\beta$ - and $\gamma$-duals of the space $\widetilde{\lambda}$ are determined, the Schauder bases for the spaces $\widetilde{c}_{1} \widetilde{c}_{0}$ and $\widetilde{\ell}_{p}$ are given, and some topological properties of the spaces $\widetilde{c}_{0}, \widetilde{\ell}_{1}$ and $\widetilde{\ell}_{p}$ are examined. Finally, the classes $\left(\widetilde{\lambda}_{1}: \lambda_{2}\right)$ and $\left(\widetilde{\lambda}_{1}: \widetilde{\lambda}_{2}\right)$ of infinite matrices are characterized, where $\lambda_{1} \in\left\{\ell_{\infty}, c_{1} c_{0}, \ell_{p}, \ell_{1}\right\}$ and $\lambda_{2} \in\left\{\ell_{\infty}, c, c_{0}, \ell_{1}\right\}$.

MSC: $46 \mathrm{~A} 45 ; 40 \mathrm{C} 05$
\end{abstract}

Keywords: matrix domain of a sequence space; $\beta$ - and $\boldsymbol{\gamma}$-duals; Schauder basis and matrix transformations

\section{Preliminaries, background and notation}

By a sequence space, we understand a linear subspace of the space $\omega=\mathbb{C}^{\mathbb{N}}$ of all complex sequences which contains $\phi$, the set of all finitely non-zero sequences, where $\mathbb{C}$ denotes the complex field and $\mathbb{N}=\{0,1,2, \ldots\}$. We write $\ell_{\infty}, c, c_{0}$ and $\ell_{p}$ for the classical sequence spaces of all bounded, convergent, null and absolutely $p$-summable sequences, respectively, where $1 \leq p<\infty$. Also, by $b s$ and $c s$, we denote the spaces of all bounded and convergent series, respectively. $b v$ is the space consisting of all sequences $\left(x_{k}\right)$ such that $\left(x_{k}-x_{k+1}\right)$ in $\ell_{1}$ and $b v_{0}$ is the intersection of the spaces $b v$ and $c_{0}$. We assume throughout, unless stated otherwise, that $p, q>1$ with $p^{-1}+q^{-1}=1$ and use the convention that any term with a negative subscript is equal to naught.

Let $A=\left(a_{n k}\right)$ be an infinite matrix of complex numbers $a_{n k}$, where $n, k \in \mathbb{N}$, and write

$$
(A x)_{n}:=\sum_{k} a_{n k} x_{k} \quad\left(n \in \mathbb{N}, x \in D_{00}(A)\right),
$$

where $D_{00}(A)$ denotes the subspace of $\omega$ consisting of $x \in \omega$ for which the sum exists as a finite sum. For simplicity in notation, here and in what follows, the summation without limits runs from 0 to $\infty$. More generally if $\mu$ is a normed sequence space, we can write $D_{\mu}(A)$ for $x \in \omega$, for which the sum in (1.1) converges in the norm of $\mu$. We write

$$
(\lambda: \mu):=\left\{A: \lambda \subseteq D_{\mu}(A)\right\}
$$

(0) 2012 Candan; licensee Springer. This is an Open Access article distributed under the terms of the Creative Commons Attribution License (http://creativecommons.org/licenses/by/2.0), which permits unrestricted use, distribution, and reproduction in any medium, provided the original work is properly cited. 
for the space of those matrices which send the whole of the sequence space $\lambda$ into the sequence space $\mu$ in this sense.

A matrix $A=\left(a_{n k}\right)$ is called a triangle if $a_{n k}=0$ for $k>n$ and $a_{n n} \neq 0$ for all $n \in \mathbb{N}$. It is trivial that $A(B x)=(A B) x$ holds for the triangle matrices $A, B$ and a sequence $x$. Further, a triangle matrix $U$ uniquely has an inverse $U^{-1}=V$ which is also a triangle matrix. Then $x=U(V x)=V(U x)$ holds for all $x \in \omega$.

Let us give the definition of some triangle limitation matrices which are needed in the text. Let $t=\left(t_{k}\right)$ be a sequence of positive reals and write

$$
T_{n}:=\sum_{k=0}^{n} t_{k} \quad(n \in \mathbb{N})
$$

Then the Cesáro mean of order one, Riesz mean with respect to the sequence $t=\left(t_{k}\right)$ and Euler mean of order $r$ are respectively defined by the matrices $C=\left(c_{n k}\right), R^{t}=\left(r_{n k}^{t}\right)$ and $E^{r}=\left(e_{n k}^{r}\right)$, where

$$
c_{n k}:=\left\{\begin{array}{ll}
\frac{1}{n+1} & (0 \leq k \leq n), \\
0 & (k>n),
\end{array} \quad r_{n k}^{t}:= \begin{cases}\frac{t_{k}}{T_{n}} & (0 \leq k \leq n), \\
0 & (k>n)\end{cases}\right.
$$

and

$$
e_{n k}^{r}:= \begin{cases}\left(\begin{array}{l}
n \\
k
\end{array}\right)(1-r)^{n-k} r^{k} & (0 \leq k \leq n), \\
0 & (k>n)\end{cases}
$$

for all $k, n \in \mathbb{N}$. We write $\mathcal{U}$ for the set of all sequences $u=\left(u_{k}\right)$ such that $u_{k} \neq 0$ for all $k \in \mathbb{N}$. For $u \in \mathcal{U}$, let $1 / u=\left(1 / u_{k}\right)$. Let $z, u, v \in \mathcal{U}$ and define the summation matrix $S=\left(s_{n k}\right)$, the difference matrix $\Delta=\left(\delta_{n k}\right)$, the generalized weighted mean or factorable matrix $G(u, v)=$ $\left(g_{n k}\right), \Delta^{(m)}=\left(\Delta_{n k}^{(m)}\right), A_{u}^{r}=\left\{a_{n k}(r)\right\}$ and $A^{z}=\left(a_{n k}^{z}\right)$ by

$$
\begin{aligned}
s_{n k} & :=\left\{\begin{array}{ll}
1 & (0 \leq k \leq n), \\
0 & (k>n),
\end{array} \quad \delta_{n k}:= \begin{cases}(-1)^{n-k} & (n-1 \leq k \leq n), \\
0 & (0 \leq k<n-1 \text { or } k>n),\end{cases} \right. \\
g_{n k}: & = \begin{cases}u_{n} v_{k} & (0 \leq k \leq n), \\
0 & (k>n),\end{cases} \\
\Delta_{n k}^{(m)}: & \begin{cases}(-1)^{n-k}\left(\begin{array}{c}
m \\
n-k
\end{array}\right) & (\max \{0, n-m\} \leq k \leq n), \\
0 & (0 \leq k<\max \{0, n-m\} \text { or } k>n),\end{cases} \\
a_{n k}(r): & \left\{\begin{array}{ll}
\frac{1+r^{k}}{n+1} u_{k} & (0 \leq k \leq n), \\
0 & (k>n)
\end{array} \text { and } a_{n k}^{z}:= \begin{cases}(-1)^{n-k} z_{k} & (n-1 \leq k \leq n), \\
0 & (0 \leq k<n-1 \text { or } k>n)\end{cases} \right.
\end{aligned}
$$

for all $k, m, n \in \mathbb{N}$, where $u_{n}$ depends only on $n$ and $v_{k}$ only on $k$. 
Let $r, s \in \mathbb{R} \backslash\{0\}$ and define the generalized difference matrix $B(r, s)=\left\{b_{n k}(r, s)\right\}$ by

$$
b_{n k}(r, s):= \begin{cases}r & (k=n), \\ s & (k=n-1), \\ 0 & (0 \leq k<n-1 \text { or } k>n)\end{cases}
$$

for all $k, n \in \mathbb{N}$. We should record here that the matrix $B(r, s)$ can be reduced to the difference matrix $\Delta^{(1)}$ in case $r=1, s=-1$. So, the results related to the matrix domain of the matrix $B(r, s)$ are more general and more comprehensive than the corresponding consequences of the matrix domain of $\Delta^{(1)}$ and include them.

The domain $\lambda_{A}$ of an infinite matrix $A$ in a sequence space $\lambda$ is defined by

$$
\lambda_{A}:=\left\{x=\left(x_{k}\right) \in \omega: A x \in \lambda\right\}
$$

which is a sequence space. If $\mathrm{A}$ is triangle, then one can easily observe that the sequence spaces $\lambda_{A}$ and $\lambda$ are linearly isomorphic, i.e., $\lambda_{A} \cong \lambda$. If $\lambda$ is a sequence space, then the continuous dual $\lambda_{A}^{*}$ of the space $\lambda_{A}$ is defined by

$$
\lambda_{A}^{*}:=\left\{f: f=g \circ A, g \in \lambda^{*}\right\}
$$

Although in most cases the new sequence space $\lambda_{A}$ generated by the limitation matrix $A$ from a sequence space $\lambda$ is the expansion or the contraction of the original space $\lambda$, it may be observed in some cases that those spaces overlap. Indeed, one can easily see that the inclusion $\lambda_{S} \subset \lambda$ strictly holds for $\lambda \in\left\{\ell_{\infty}, c, c_{0}\right\}$. Similarly, one can deduce that the inclusion $\lambda \subset \lambda_{\Delta^{(1)}}$ also strictly holds for $\lambda \in\left\{\ell_{\infty}, c, c_{0}, \ell_{p}\right\}$. However, if we define $\lambda:=c_{0} \oplus$ $\operatorname{span}\{z\}$ with $z=\left((-1)^{k}\right)$, i.e., $x \in \lambda$ if and only if $x:=s+\alpha z$ for some $s \in c_{0}$ and some $\alpha \in \mathbb{C}$, and consider the matrix $A$ with the rows $A_{n}$ defined by $A_{n}:=(-1)^{n} e^{(n)}$ for all $n \in \mathbb{N}$, we have $A e=z \in \lambda$ but $A z=e \notin \lambda$ which lead us to the consequences that $z \in \lambda \backslash \lambda_{A}$ and $e \in \lambda_{A} \backslash \lambda$, where $e=(1,1,1, \ldots)$ and $e^{(n)}$ is a sequence whose only non-zero term is a 1 in $n$th place for each $n \in \mathbb{N}$. That is to say, the sequence spaces $\lambda_{A}$ and $\lambda$ overlap but neither contains the other. The approach of constructing a new sequence space by means of the matrix domain of a particular limitation method has recently been employed by Wang [1], Ng and Lee [2], Malkowsky [4], Altay and Başar [10, 20, 36, 37, 41, 46], Malkowsky and Savaş [8], Başarır [47], Aydın and Başar [12, 13, 16, 30, 39], Başar et al. [38], Şengönül and Başar [9], Altay [23], Polat and Başar [25] and Malkowsky et al. [43]. In Table $1, \Delta, \Delta^{2}$ and $\Delta^{m}$ are the transpose of the matrices $\Delta^{(1)}, \Delta^{(2)}$ and $\Delta^{(m)}$, respectively, and $c_{0}(u, p)$ and $c(u, p)$ are the spaces consisting of the sequences $x=\left(x_{k}\right)$ such that $u x=\left(u_{k} x_{k}\right)$ in the spaces $c_{0}(p)$ and $c(p)$ for $u \in \mathcal{U}$, respectively, and studied by Başarır [47]. Finally, the new technique for deducing certain topological properties, for example $A B-, K B-, A D$-properties, solidity and monotonicity etc., and determining the $\beta$ - and $\gamma$-duals of the domain of a triangle matrix in a sequence space is given by Altay and Başar [46]. 
Table 1 The domains of some triangle matrices in certain sequence spaces

\begin{tabular}{|c|c|c|c|}
\hline$\lambda$ & $\boldsymbol{A}$ & $\lambda_{A}$ & Refer to: \\
\hline c & $N_{q}$ & $C_{N q}$ & [1] \\
\hline$\ell_{p},(1 \leq p \leq \infty)$ & C & $x_{p}, x_{\infty}$ & [2] \\
\hline$x_{p},(1 \leq p \leq \infty)$ & $\Delta^{m}$ & $C_{p}\left(\Delta^{m}\right), C_{\infty}\left(\Delta^{m}\right)$ & [3] \\
\hline$c_{0}, c_{1} \ell_{\infty}$ & $R^{q}$ & $(\bar{N}, q)_{0},(\bar{N}, q)_{,}(\bar{N}, q)_{\infty}$ & [4] \\
\hline$c_{0}, c_{,} \ell_{\infty}$ & $\Delta^{(1)}$ & $c_{0}(\Delta), c(\Delta), \ell_{\infty}(\Delta)$ & [5] \\
\hline$c_{0}, c_{1} \ell_{\infty}$ & $\Delta^{2}$ & $c_{0}\left(\Delta^{2}\right), c\left(\Delta^{2}\right), \ell_{\infty}\left(\Delta^{2}\right)$ & [6] \\
\hline$c_{0}, c_{1} \ell_{\infty}$ & $u \Delta^{2}$ & $c_{0}\left(u ; \Delta^{2}\right), c\left(u ; \Delta^{2}\right), \ell_{\infty}\left(u ; \Delta^{2}\right)$ & [7] \\
\hline$c_{0}, c_{1} l_{\infty}$ & $\Delta^{2}$ & $c_{0}\left(\Delta^{2}\right), c\left(\Delta^{2}\right), \ell_{\infty}\left(\Delta^{2}\right)$ & [6] \\
\hline$c_{0}, c_{1} \ell_{p}$ & $G(u, v)$ & $Z\left(u, v ; c_{0}\right), Z(u, v ; c), Z\left(u, v ; \ell_{p}\right)$ & [8] \\
\hline$c_{0}, c$ & C & $\tilde{c}_{0}, \tilde{c}$ & [9] \\
\hline$c_{0, c}$ & $E^{r}$ & $e_{0}^{r}, e_{c}^{r}$ & [10] \\
\hline$c_{0, c}$ & $G(u, v)$ & 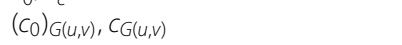 & [11] \\
\hline$c_{0}, c$ & $A_{1}^{r}$ & $a_{0}^{r}, a_{c}^{r}$ & [12] \\
\hline$\ell_{p},(1 \leq p \leq \infty)$ & $A_{1}^{r}$ & $a_{p}^{r}, a_{\infty}^{r}$ & [13] \\
\hline$\ell_{p},(1 \leq p \leq \infty)$ & $E^{r}$ & $e_{p^{\prime}}^{r} e_{\infty}^{r}$ & {$[14,15]$} \\
\hline$a_{0}^{r}, a_{c}^{r}$ & $\Delta^{(1)}$ & $a_{0}^{r}(\Delta), a_{c}^{r}(\Delta)$ & {$[16]$} \\
\hline$\ell_{p^{\prime}}(1 \leq p<\infty)$ & $G(u, v)$ & $\ell_{A}^{p}$ & {$[17]$} \\
\hline$\ell_{p},(1 \leq p<\infty)$ & $\Delta^{(1)}$ & $b v_{p}$ & {$[18,19]$} \\
\hline$\ell_{p},(0<p<1)$ & $\Delta^{(1)}$ & $b v_{p}$ & {$[20]$} \\
\hline$c_{0}, c_{1} \ell_{\infty}$ & $\Delta^{m}$ & $c_{0}\left(\Delta^{m}\right), c\left(\Delta^{m}\right), \ell_{\infty}\left(\Delta^{m}\right)$ & {$[21,22]$} \\
\hline$\ell_{p},(1 \leq p<\infty)$ & $\Delta^{(m)}$ & $\ell_{p}\left(\Delta^{(m)}\right)$ & [23] \\
\hline$c_{0}, c_{1} \ell_{\infty}$ & $\Delta^{(m)}$ & $c_{0}\left(\Delta^{(m)}\right), c\left(\Delta^{(m)}\right), \ell_{\infty}\left(\Delta^{(m)}\right)$ & [24] \\
\hline$e_{0}^{r}, e_{c}^{r}$ & $\Delta^{(m)}$ & $e_{0}^{r}\left(\Delta^{(m)}\right), e_{c}^{r}\left(\Delta^{(m)}\right)$ & [25] \\
\hline$w_{0}^{p}, w^{p}, w_{\infty}^{p}$ & $\Delta$ & $w_{0}^{p}(\Delta), w^{p}(\Delta), w_{\infty}^{p}(\Delta)$ & [26] \\
\hline$w_{0}^{p}, w^{p}, w_{\infty}^{p}$ & $T$ & $w_{0}^{p}(T), w^{p}(T), w_{\infty}^{p}(T)$ & {$[27]$} \\
\hline$\ell_{\infty}(p)$ & $S$ & $b s(p)$ & {$[28,29]$} \\
\hline$\ell(p)$ & $A_{u}^{r}$ & $a^{r}(u, p)$ & {$[30]$} \\
\hline$\ell(p)$ & $B(r, s)$ & $\widehat{\ell}(p)$ & [31] \\
\hline$\ell(p)$ & S & $\overline{\ell(p)}$ & [32] \\
\hline$c_{0}(p), c(p), \ell_{\infty}(p)$ & $\Delta$ & $\Delta c_{0}(p), \Delta c(p), \Delta \ell_{\infty}(p)$ & [33] \\
\hline$c_{0}(p), c(p), \ell_{\infty}(p)$ & $u \Delta$ & $c_{0}(u, \Delta, p), c(u, \Delta, p), \ell_{\infty}(u, \Delta, p)$ & [34] \\
\hline$c_{0}(p), c(p), \ell_{\infty}(p)$ & $u \Delta^{2}$ & $c_{0}\left(u, \Delta^{2}, p\right), c\left(u, \Delta^{2}, p\right), \ell_{\infty}\left(u, \Delta^{2}, p\right)$ & {$[35]$} \\
\hline$c_{0}(p), c(p), \ell_{\infty}(p)$ & $G(u, v)$ & $c_{0}(u, v ; p), c(u, v ; p), \ell_{\infty}(u, v ; p)$ & [36] \\
\hline$\ell(p)$ & $G(u, v)$ & $\ell(u, v ; p)$ & [37] \\
\hline$\ell(p), \ell_{\infty}(p)$ & $A^{z}$ & $b v(z, p), b v_{\infty}(z, p)$ & [38] \\
\hline$c_{0}(u, p), c(u, p)$ & $A_{1}^{r}$ & $a_{0}^{r}(u, p), a_{c}^{r}(u, p)$ & [39] \\
\hline$\ell(p)$ & $R^{t}$ & $r^{t}(p)$ & {$[40]$} \\
\hline$c_{0}(p), c(p), \ell_{\infty}(p)$ & $R^{t}$ & $r_{0}^{t}(p), r_{c}^{t}(p), r_{\infty}^{t}(p)$ & [41] \\
\hline$c_{0}(p), c(p), \ell_{\infty}(p)$ & $\Delta^{m}$ & $\Delta^{m} c_{0}(p), \Delta^{m} c(p), \Delta^{m} \ell_{\infty}(p)$ & {$[42]$} \\
\hline$c_{0}(p), c(p), \ell_{\infty}(p)$ & $u \Delta^{(m)}$ & $\Delta_{u}^{(m)} c_{0}(p), \Delta_{u}^{(m)} c(p), \Delta_{u}^{(m)} \ell_{\infty}(p)$ & [43] \\
\hline$c_{0}, c_{1} \ell_{\infty}, \ell_{p}$ & $B(r, s)$ & $\hat{c}_{0}, \hat{c}_{,} \hat{l}_{\infty}, \hat{l}_{p}$ & [44] \\
\hline$c_{0}, c_{,} \ell_{\infty}, \ell_{p}$ & $B(r, s, t)$ & $c_{0}(B), C(B), \ell_{\infty}(B), \ell_{p}(B)$ & [45] \\
\hline
\end{tabular}

Let $\tilde{r}=\left(r_{n}\right)_{n=0}^{\infty}$ and $\tilde{s}=\left(s_{n}\right)_{n=0}^{\infty}$ be given convergent sequences of positive real numbers. Define the sequential generalized difference matrix $B(\widetilde{r}, \widetilde{s})=\left\{b_{n k}(\widetilde{r}, \widetilde{s})\right\}$ by

$$
b_{n k}(\widetilde{r}, \widetilde{s}):= \begin{cases}r_{n} & (k=n), \\ s_{n} & (k=n-1), \\ 0 & (0 \leq k<n-1 \text { or } k>n),\end{cases}
$$

for all $k, n \in \mathbb{N}$, the set of natural numbers. We should record here that the matrix $B(\widetilde{r}, \widetilde{s})$ can be reduced to the generalized difference matrix $B(r, s)$ in the case $r_{n}=r$ and $s_{n}=s$ for all $n \in \mathbb{N}$. So, the results related to the matrix domain of the matrix $B(\tilde{r}, \tilde{s})$ are more general and more comprehensive than the corresponding consequences of the matrix domain of 
$B(r, s)$ and include them. For the literature concerning the domain $\lambda_{A}$ of the infinite matrix $A$ in the sequence space $\lambda$, Table 1 may be useful.

The main purpose of the present paper is to introduce the sequence space $\lambda_{B(\widetilde{r}, \widetilde{s})}$ and to determine the $\beta$-and $\gamma$-duals of the space, where $\lambda$ denotes any one of the spaces $\ell_{\infty}, c$, $c_{0}$ or $\ell_{p}$. Furthermore, the Schauder bases for the spaces $\tilde{c}, \widetilde{c}_{0}$ and $\tilde{\ell}_{p}$ are given and some topological properties of the spaces $\widetilde{c}_{0}, \widetilde{\ell}_{1}$ and $\tilde{\ell}_{p}$ are examined. Finally, some classes of matrix mappings on the space $\lambda_{B(\tilde{r}, \widetilde{S})}$ are characterized.

The paper is organized as follows. In Section 2, we summarize the studies on the difference sequence spaces. In Section 3, we introduce the domain $\lambda_{B(r, \widetilde{s})}$ of the generalized difference matrix $B(\widetilde{r}, \widetilde{s})$ in the sequence space $\lambda$ with $\lambda \in\left\{\ell_{\infty}, c, c_{0}, \ell_{p}\right\}$ and determine the $\beta$ - and $\gamma$-duals of $\lambda_{B(r, s)}$. After proving the fact, under which conditions for the inclusion $\lambda \subset \lambda_{B(\tilde{r}, \tilde{s})}$ and the equality $\lambda=\lambda_{B(r, \widetilde{s})}$ hold, we give the Schauder basis of the spaces $\left(c_{0}\right)_{B(\tilde{r}, \tilde{s})}, c_{B(\tilde{r}, \tilde{s})}$ and $\left(\ell_{p}\right)_{B(\tilde{r}, \tilde{s})}$. Finally, we investigate some topological properties of the spaces $\left(c_{0}\right)_{B(\widetilde{r}, \widetilde{s}},\left(\ell_{1}\right)_{B(\tilde{r}, \widetilde{s}}$ and $\left(\ell_{p}\right)_{B(\widetilde{r}, \widetilde{s})}$ with $p>1$. In Section 4 , we state and prove a general theorem characterizing the matrix transformations from the domain of a triangle matrix to any sequence space. As an application of this basic theorem, we make a table which gives the necessary and sufficient conditions of the matrix transformations from $\lambda_{B(\tilde{r}, \tilde{s})}$ to $\mu$, where $\lambda \in\left\{\ell_{\infty}, c, c_{0}, \ell_{p}\right\}$ and $\mu \in\left\{\ell_{\infty}, c, c_{0}, \ell_{1}\right\}$. In the final section of the paper, we note the significance of the present results in the literature about difference sequences and record some further suggestions.

\section{Difference sequence spaces}

In this section, we give some knowledge about the literature concerning the spaces of difference sequences.

Let $\lambda$ denote any one of the classical sequence spaces $\ell_{\infty}, c$ or $c_{0}$. Then $\lambda(\Delta)$ consisting of the sequences $x=\left(x_{k}\right)$ such that $\Delta x=\left(x_{k}-x_{k+1}\right) \in \lambda$ is called the difference sequence spaces which were introduced by Kızmaz [5]. Kızmaz [5] proved that $\lambda(\Delta)$ is a Banach space with the norm

$$
\|x\|_{\Delta}=\left|x_{1}\right|+\|\Delta x\|_{\infty} ; \quad x=\left(x_{k}\right) \in \lambda(\Delta)
$$

and the inclusion relation $\lambda \subset \lambda(\Delta)$ strictly holds. He also determined the $\alpha$-, $\beta$ - and $\gamma$ duals of the difference spaces and characterized the classes $(\lambda(\Delta): \mu)$ and $(\mu: \lambda(\Delta))$ of infinite matrices, where $\lambda, \mu \in\left\{\ell_{\infty}, c\right\}$. Following Kızmaz [5], Sarıgöl [48] extended the difference spaces $\lambda(\Delta)$ to the spaces $\lambda\left(\Delta_{r}\right)$ defined by

$$
\lambda\left(\Delta_{r}\right):=\left\{x=\left(x_{k}\right) \in \omega: \Delta_{r} x=\left\{k^{r}\left(x_{k}-x_{k+1}\right)\right\} \in \lambda \text { for } r<1\right\}
$$

and computed the $\alpha-, \beta-, \gamma$-duals of the space $\lambda\left(\Delta_{r}\right)$, where $\lambda \in\left\{\ell_{\infty}, c, c_{0}\right\}$. It is easily seen that $\lambda\left(\Delta_{r}\right) \subset \lambda(\Delta)$, if $0<r<1$ and $\lambda(\Delta) \subset \lambda\left(\Delta_{r}\right)$, if $r<0$.

In the same year, Ahmad and Mursaleen [33] extended these spaces to $\lambda(p, \Delta)$ and studied related problems. Malkowsky [49] determined the Köthe-Toeplitz duals of the sets $\ell_{\infty}(p, \Delta)$ and $c_{0}(p, \Delta)$ and gave new proofs of the characterization of the matrix transformations considered in [33]. In 1993, Choudhary and Mishra [50] studied some properties of the sequence space $c_{0}\left(\Delta_{r}\right)$ for $r \geq 1$. In the same year, Mishra [51] gave a characterization of $B K$-spaces which contain a subspace isomorphic to $s c_{0}(\Delta)$ in terms of matrix maps and a sufficient condition for a matrix map from $s \ell_{\infty}(\Delta)$ into a $B K$-space to be a compact 
operator. He showed that any matrix from $s \ell_{\infty}(\Delta)$ into a $B K$-space which does not contain any subspace isomorphic to $s \ell_{\infty}(\Delta)$ is compact, where

$$
s \lambda(\Delta)=\left\{x=\left(x_{k}\right) \in \omega:\left(\Delta x_{k}\right) \in \lambda, x_{1}=0 \text { for } \lambda=\ell_{\infty} \text { or } c_{0}\right\} .
$$

In 1996, Mursaleen et al. [52] defined and studied the sequence space

$$
\ell_{\infty}\left(p, \Delta_{r}\right)=\left\{x=\left(x_{k}\right) \in \omega: \Delta_{r} x \in \ell_{\infty}(p)\right\} \quad(r>0) .
$$

Gnanaseelan and Srivastava [53] defined and studied the spaces $\lambda(u, \Delta)$ for a sequence $u=\left(u_{k}\right)$ of non-complex numbers such that

(i) $\frac{\left|u_{k}\right|}{\left|u_{k+1}\right|}=1+O(1 / k)$ for each $k \in \mathbb{N}_{1}=\{1,2,3, \ldots\}$.

(ii) $k^{-1}\left|u_{k}\right| \sum_{i=0}^{k}\left|u_{i}\right|^{-1}=O(1)$.

(iii) $\left(k\left|u_{k}^{-1}\right|\right)$ is a sequence of positive numbers increasing monotonically to infinity.

In the same year, Malkowsky [54] defined the spaces $\lambda(u, \Delta)$ for an arbitrary fixed sequence $u=\left(u_{k}\right)$ without any restrictions on $u$. He proved that the sequence spaces $\lambda(u, \Delta)$ are $B K$-spaces with the norm defined by

$$
\|x\|=\sup _{k \in \mathbb{N}}\left|u_{k-1}\left(x_{k-1}-x_{k}\right)\right| \quad \text { with } u_{0}=x_{0}=1
$$

Later, Gaur and Mursaleen [55] extended the space $S_{r}(\Delta)$ to the space $S_{r}(p, \Delta)$, where

$$
S_{r}(p, \Delta)=\left\{x=\left(x_{k}\right) \in \omega:\left(k^{r}\left|\Delta x_{k}\right|\right) \in c_{0}(p)\right\} \quad(r \geq 1)
$$

and characterized the matrix classes $\left(S_{r}(p, \Delta): \ell_{\infty}\right)$ and $\left(S_{r}(p, \Delta): \ell_{1}\right)$. Malkowsky et al. [56] and, independently, Asma and Çolak [34] extended the space $\lambda(u, \Delta)$ to the space $\lambda(p, u, \Delta)$ and gave Köthe-Toeplitz duals of this spaces for $\lambda=\ell_{\infty}, c$ or $c_{0}$. Recently, Malkowsky and Mursaleen [57] characterized the matrix classes $(\Delta \lambda: \mu)$ and $(\Delta \lambda: \Delta \mu)$ for $\lambda=c_{0}(p), c(p), \ell_{\infty}(p)$ and $\mu=c_{0}(q), c(q), \ell_{\infty}(q)$.

Recently, the difference spaces $b v_{p}$ consisting of the sequences $x=\left(x_{k}\right)$ such that $\left(x_{k}-\right.$ $\left.x_{k-1}\right) \in \ell_{p}$ have been studied in the case $0<p<1$ by Altay and Başar [20], and in the case $1 \leq p \leq \infty$ by Başar and Altay [18] and Çolak et al. [19].

\section{Some new sequence spaces derived by the domain of the matrix $B(\tilde{r}, \tilde{s})$}

In this section, we define the sequence spaces $\tilde{\ell}_{\infty}, \widetilde{c}, \widetilde{c}_{0}$ and $\tilde{\ell}_{p}$, and determine the $\beta$ - and $\gamma$-duals of the spaces.

We introduce the sequence spaces $\tilde{\ell}_{\infty}, \widetilde{c}, \widetilde{c}_{0}$ and $\tilde{\ell}_{p}$ as the set of all sequences whose $B(\widetilde{r}, \widetilde{s})$-transforms are in the spaces $\ell_{\infty}, c, c_{0}$ and $\ell_{p}$, respectively, that is,

$$
\begin{aligned}
& \tilde{\ell}_{\infty}:=\left\{x=\left(x_{k}\right) \in \omega: \sup _{k \in \mathbb{N}}\left|s_{k-1} x_{k-1}+r_{k} x_{k}\right|<\infty\right\}, \\
& \tilde{c}:=\left\{x=\left(x_{k}\right) \in \omega: \exists l \in \mathbb{C} \ni \lim _{k \rightarrow \infty}\left|s_{k-1} x_{k-1}+r_{k} x_{k}-l\right|=0\right\}, \\
& \tilde{c}_{0}:=\left\{x=\left(x_{k}\right) \in \omega: \lim _{k \rightarrow \infty}\left|s_{k-1} x_{k-1}+r_{k} x_{k}\right|=0\right\}, \\
& \tilde{\ell}_{p}:=\left\{x=\left(x_{k}\right) \in \omega: \sum_{k}\left|s_{k-1} x_{k-1}+r_{k} x_{k}\right|^{p}<\infty\right\} .
\end{aligned}
$$


With the notation of (1.2), we can redefine the spaces $\tilde{\ell}_{\infty}, \widetilde{c}, \widetilde{c}_{0}$ and $\tilde{\ell}_{p}$ by

$$
\tilde{\ell}_{\infty}:=\left\{\ell_{\infty}\right\}_{B(\tilde{r}, \tilde{s})}, \quad \widetilde{c}:=c_{B(\tilde{r}, \tilde{s})}, \quad \widetilde{c}_{0}:=\left\{c_{0}\right\}_{B(\tilde{r}, \tilde{s})}, \quad \tilde{\ell}_{p}:=\left\{\ell_{p}\right\}_{B(\tilde{r}, \tilde{s})}
$$

Define the sequence $y=\left(y_{k}\right)$ by the $B(\widetilde{r}, \widetilde{s})$-transform of a sequence $x=\left(x_{k}\right)$, i.e.,

$$
y_{k}:=s_{k-1} x_{k-1}+r_{k} x_{k} \quad(k \in \mathbb{N}) .
$$

Since the spaces $\lambda$ and $\lambda_{B(\widetilde{r}, \mathcal{S})}$ are linearly isomorphic, one can easily observe that $x=\left(x_{k}\right) \in$ $\lambda_{B(\tilde{r}, \tilde{s})}$ if and only if $y=\left(y_{k}\right) \in \lambda$, where the sequences $x=\left(x_{k}\right)$ and $y=\left(y_{k}\right)$ are connected with the relation (3.1).

Prior to quoting the lemmas which are needed for deriving some consequences given in Corollary 3.4 below, we give an inclusion theorem related to these new spaces.

Theorem 3.1 Let $\lambda \in\left\{\ell_{\infty}, c, c_{0}, \ell_{p}\right\}$ and $B=B(\tilde{r}, \tilde{s})$. Then

(i) $\lambda=\lambda_{B}$, if $\frac{\sup s_{n}}{\inf r_{n}}<1$.

(ii) $\lambda \subset \lambda_{B}$ is strict, if $\frac{\sup s_{n}}{\inf r_{n}} \geq 1$.

Proof Let $\lambda \in\left\{\ell_{\infty}, c, c_{0}, \ell_{1}\right\}$ and $B=B(\tilde{r}, \tilde{s})$. Since the matrix $B$ satisfies the conditions

$$
\begin{aligned}
& \sup _{n \in \mathbb{N}} \sum_{k}\left|b_{n k}\right| \leq \sup _{n \in \mathbb{N}} r_{n}+\sup _{n \in \mathbb{N}} s_{n}, \\
& \lim _{n \rightarrow \infty} b_{n k}=0, \\
& \lim _{n \rightarrow \infty} \sum_{k} b_{n k}=\lim _{n \rightarrow \infty} r_{n}+\lim _{n \rightarrow \infty} s_{n}
\end{aligned}
$$

and

$$
\sup _{k \in \mathbb{N}} \sum_{n}\left|b_{n k}\right| \leq \sup _{k \in \mathbb{N}} r_{k}+\sup _{k \in \mathbb{N}} s_{k}
$$

$B \in(\lambda: \lambda)$. For any sequence $x \in \lambda, B x \in \lambda$ hence $x \in \lambda_{B}$. This shows that $\lambda \subset \lambda_{B}$.

(i) Let $\frac{\sup s_{n}}{\inf r_{n}}<1$. Since the inverse matrix $B^{-1}=\left(b_{n k}^{-1}\right)$ of the matrix $B$ also satisfies the conditions

$$
\begin{aligned}
& \sup _{n \in \mathbb{N}} \sum_{k}\left|b_{n k}^{-1}\right| \leq \frac{1}{\inf r_{n}} \sum_{k}\left(\frac{\sup s_{n}}{\inf r_{n}}\right)^{k}<\infty, \\
& \lim _{n \rightarrow \infty} b_{n k}^{-1}=\lim _{n \rightarrow \infty} \frac{1}{r_{n}} \prod_{i=k}^{n-1} \frac{-s_{i}}{r_{i}}=0, \\
& \lim _{n \rightarrow \infty} \sum_{k} b_{n k}^{-1} \leq \frac{1}{\inf r_{n}} \lim _{n \rightarrow \infty} \sum_{k=0}^{n}\left(-\frac{\inf s_{n}}{\inf r_{n}}\right)^{k} \text { exists }
\end{aligned}
$$

and

$$
\sup _{k \in \mathbb{N}} \sum_{k}\left|b_{n k}^{-1}\right| \leq \frac{1}{\inf r_{k}} \sum_{n}\left(\frac{\sup s_{k}}{\inf r_{k}}\right)^{n}<\infty
$$


$B^{-1} \in(\lambda: \lambda)$. Therefore, if $x \in \lambda_{B}$, then $y=B x \in \lambda$ and $x=B^{-1} y \in \lambda$. Thus, the opposite inclusion $\lambda_{B} \subset \lambda$ is just proved. This completes the proof of Part (i).

(ii) Let us consider the sequences $u^{1}:=\left\{\frac{1}{r_{n}} \prod_{i=0}^{n-1}-\frac{s_{i}}{r_{i}}\right\}, u^{2}:=\left\{(-1)^{n}(n+1)\right\}$ and $u^{3}:=\{[1+$ $\left.\left.(-1)^{n}\right] / 2\right\}$.

If $\frac{\sup s_{n}}{\inf r_{n}}>1$, then $B u^{1}=e^{(0)}=(1,0,0, \ldots) \in \lambda$. Hence, $u^{1} \in \lambda_{B} \backslash \lambda$.

Suppose that $\frac{\sup s_{n}}{\inf r_{n}}=1$.

(a) Let $\lambda=c_{0}, \ell_{p}$. If $\left(s_{n}\right)=\left(r_{n}\right)$, then $u^{1} \in \lambda_{B} \backslash \lambda$.

(b) Let $\lambda=\ell_{\infty}, c$. If $\left(s_{n}\right)=\left(r_{n+1}\right)$, then $B u^{2}=\left\{r_{n}(-1)^{n}\right\} \in \ell_{\infty}, B u^{3}=\left(r_{0}, r_{1}, r_{2}, \ldots\right) \in c$.

Hence, $u^{2} \in\left(\ell_{\infty}\right)_{B} \backslash \ell_{\infty}$ and $u^{3} \in c_{B} \backslash c$.

This step completes the proof.

The set $S(\lambda, \mu)$ defined by

$$
S(\lambda, \mu):=\left\{z=\left(z_{k}\right) \in \omega: x z=\left(x_{k} z_{k}\right) \in \mu \text { for all } x=\left(x_{k}\right) \in \lambda\right\}
$$

is called the multiplier space of the spaces $\lambda$ and $\mu$. One can easily observe for a sequence space $v$ with $\lambda \supset v \supset \mu$ that the inclusions

$$
S(\lambda, \mu) \subset S(\nu, \mu) \text { and } S(\lambda, \mu) \subset S(\lambda, \nu)
$$

hold. With the notation of (3.2), the $\alpha-, \beta$ - and $\gamma$-duals of a sequence space $\lambda$, which are respectively denoted by $\lambda^{\alpha}, \lambda^{\beta}$ and $\lambda^{\gamma}$, are defined by

$$
\lambda^{\alpha}:=S\left(\lambda, \ell_{1}\right), \quad \lambda^{\beta}:=S(\lambda, c s) \quad \text { and } \quad \lambda^{\gamma}:=S(\lambda, b s)
$$

Lemma $3.2[58$, p.52, Exercise 2.5(i)] Let $\lambda, \mu$ be the sequence spaces and $\xi \in\{\alpha, \beta, \gamma\}$. If $\lambda \subset \mu$, then $\mu^{\xi} \subset \lambda^{\xi}$.

We read the following useful results from Stieglitz and Tietz [59]:

$$
\begin{aligned}
& \sup _{n \in \mathbb{N}} \sum_{k}\left|a_{n k}\right|^{q}<\infty, \\
& \sup _{k, n \in \mathbb{N}}\left|a_{n k}\right|<\infty, \\
& \lim _{n \rightarrow \infty} a_{n k}=\alpha_{k} \quad(k \in \mathbb{N}), \\
& \lim _{n \rightarrow \infty} \sum_{k}\left|a_{n k}\right|=\sum_{k}\left|\alpha_{k}\right|, \\
& \lim _{n \rightarrow \infty} \sum_{k} a_{n k}=\alpha .
\end{aligned}
$$

Lemma 3.3 The necessary and sufficient conditions for $A \in(\lambda: \mu)$ when $\lambda \in\left\{\ell_{\infty}, c, c_{0}, \ell_{1}\right.$, $\left.\ell_{p}\right\}$ and $\mu \in\left\{\ell_{\infty}, c\right\}$ can be read from Table 2 .

Basic Lemma [46, Theorem 3.1] Let $C=\left(c_{n k}\right)$ be defined via the sequence $a=\left(a_{k}\right) \in \omega$ and the inverse matrix $V=\left(v_{n k}\right)$ of the triangle matrix $U=\left(u_{n k}\right)$ by

$$
c_{n k}:= \begin{cases}\sum_{j=k}^{n} a_{j} v_{j k} & (0 \leq k \leq n), \\ 0 & (k>n)\end{cases}
$$


Table 2 The characterization of the class $\left(\lambda_{1}: \lambda_{2}\right)$ with $\lambda_{1} \in\left\{\ell_{\infty}, c, c_{0}, \ell_{p}, \ell_{1}\right\}$ and $\lambda_{2} \in\left\{\ell_{\infty}, c\right\}$

\begin{tabular}{llllll}
\hline To & \multicolumn{3}{l}{ From } & & \\
\cline { 2 - 6 } & $\boldsymbol{\ell}_{\infty}$ & $\boldsymbol{c}$ & $\boldsymbol{c}_{\mathbf{0}}$ & $\boldsymbol{\ell}_{\boldsymbol{p}}$ & $\boldsymbol{\ell}_{\mathbf{1}}$ \\
\hline $\boldsymbol{\ell}_{\infty}$ & 1. & 1. & 1. & 2. & 3. \\
$c$ & 4. & 5. & 6. & 7. & 8. \\
\hline
\end{tabular}

Here 1. (3.3) with $q=1$. 2. (3.3). 3. (3.4). 4. (3.5) and (3.6). 5. (3.3) with $q=1$, (3.5) and (3.7). 6. (3.3) with $q=1$ and (3.5). 7. (3.3) and (3.5). 8. (3.4) and (3.5).

for all $k, n \in \mathbb{N}$. Then

$$
\left\{\lambda_{U}\right\}^{\gamma}:=\left\{a=\left(a_{k}\right) \in \omega: C \in\left(\lambda: \ell_{\infty}\right)\right\}
$$

and

$$
\left\{\lambda_{U}\right\}^{\beta}:=\left\{a=\left(a_{k}\right) \in \omega: C \in(\lambda: c)\right\} .
$$

Combining Lemma 3.3 with Basic Lemma, we have

Corollary 3.4 Define the sets $d_{1}(\widetilde{r}, \widetilde{s}), d_{2}(\tilde{r}, \tilde{s}), d_{3}(\tilde{r}, \widetilde{s}), d_{4}(\widetilde{r}, \widetilde{s})$ and $d_{5}(\tilde{r}, \widetilde{s})$ by

$$
\begin{aligned}
& d_{1}(\widetilde{r}, \widetilde{s}):=\left\{a=\left(a_{k}\right) \in \omega: \sup _{n \in \mathbb{N}} \sum_{k=0}^{n}\left|\sum_{j=k}^{n} \frac{1}{r_{j}} \prod_{i=k}^{j-1} \frac{-s_{i}}{r_{i}} a_{j}\right|^{q}<\infty\right\}, \\
& d_{2}(\widetilde{r}, \widetilde{s}):=\left\{a=\left(a_{k}\right) \in \omega: \lim _{n \rightarrow \infty} \sum_{j=k}^{n} \frac{1}{r_{j}} \prod_{i=k}^{j-1} \frac{-s_{i}}{r_{i}} a_{j} \text { exists }\right\}, \\
& d_{3}(\widetilde{r}, \widetilde{s}):=\left\{a=\left(a_{k}\right) \in \omega: \lim _{n \rightarrow \infty} \sum_{k=0}^{n}\left|\sum_{j=k}^{n} \frac{1}{r_{j}} \prod_{i=k}^{j-1} \frac{-s_{i}}{r_{i}} a_{j}\right|=\sum_{k=0}^{\infty}\left|\lim _{n \rightarrow \infty} \sum_{j=k}^{n} \frac{1}{r_{j}} \prod_{i=k}^{j-1} \frac{-s_{i}}{r_{i}} a_{j}\right|\right\}, \\
& d_{4}(\widetilde{r}, \widetilde{s}):=\left\{a=\left(a_{k}\right) \in \omega: \lim _{n \rightarrow \infty} \sum_{k=0}^{n} \sum_{j=k}^{n} \frac{1}{r_{j}} \prod_{i=k}^{j-1} \frac{-s_{i}}{r_{i}} a_{j} \text { exists }\right\},
\end{aligned}
$$

and

$$
d_{5}(\widetilde{r}, \widetilde{s}):=\left\{a=\left(a_{k}\right) \in \omega: \sup _{k, n \in \mathbb{N}}\left|\sum_{j=k}^{n} \frac{1}{r_{j}} \prod_{i=k}^{j-1} \frac{-s_{i}}{r_{i}} a_{j}\right|<\infty\right\} .
$$

Then

(i) $\left\{\widetilde{\ell}_{\infty}\right\}^{\gamma}:=\widetilde{c}^{\gamma}:=\left\{\widetilde{c}_{0}\right\}^{\gamma}:=d_{1}(\tilde{r}, \widetilde{s})$ with $q=1$.

(ii) $\left\{\tilde{\ell}_{p}\right\}^{\gamma}:=d_{1}(\widetilde{r}, \widetilde{s})$.

(iii) $\left\{\tilde{\ell}_{1}\right\}^{\gamma}:=d_{5}(\widetilde{r}, \widetilde{s})$.

(iv) $\left\{\tilde{\ell}_{\infty}\right\}^{\beta}:=d_{2}(\tilde{r}, \tilde{s}) \cap d_{3}(\tilde{r}, \widetilde{s})$.

(v) $\widetilde{c}^{\beta}:=d_{1}(\tilde{r}, \widetilde{s}) \cap d_{2}(\widetilde{r}, \widetilde{s}) \cap d_{4}(\widetilde{r}, \widetilde{s})$ with $q=1$.

(vi) $\left\{\widetilde{c}_{0}\right\}^{\beta}:=d_{1}(\widetilde{r}, \widetilde{s}) \cap d_{2}(\widetilde{r}, \widetilde{s})$ with $q=1$.

(vii) $\left\{\tilde{\ell}_{p}\right\}^{\beta}:=d_{1}(\widetilde{r}, \widetilde{s}) \cap d_{2}(\widetilde{r}, \widetilde{s})$.

(viii) $\left\{\widetilde{\ell}_{1}\right\}^{\beta}:=d_{2}(\tilde{r}, \tilde{s}) \cap d_{5}(\widetilde{r}, \widetilde{s})$. 
A sequence space $\lambda$ with a linear topology is called a $K$-space provided each of the maps $p_{i}: \lambda \rightarrow \mathbb{C}$ defined by $p_{i}(x)=x_{i}$ is continuous for all $i \in \mathbb{N}$. A $K$-space $\lambda$ is called an $F K$-space provided $\lambda$ is a complete linear metric space. An $F K$-space whose topology is normable is called a $B K$-space. If a normed sequence space $\lambda$ contains a sequence $\left(b_{n}\right)$ with the property that for every $x \in \lambda$, there is a unique sequence of scalars $\left(\alpha_{n}\right)$ such that

$$
\lim _{n \rightarrow \infty}\left\|x-\left(\alpha_{0} b_{0}+\alpha_{1} b_{1}+\cdots+\alpha_{n} b_{n}\right)\right\|=0,
$$

then $\left(b_{n}\right)$ is called a Schauder basis (or briefly basis) for $\lambda$. The series $\sum \alpha_{k} b_{k}$ which has the sum $x$ is then called the expansion of $x$ with respect to $\left(b_{n}\right)$ and written as $x=\sum \alpha_{k} b_{k}$.

Since it is known that the matrix domain $\lambda_{A}$ of a normed sequence space $\lambda$ has a basis if and only if $\lambda$ has a basis whenever $A=\left(a_{n k}\right)$ is a triangle (cf. [60, Remark 2.4]), we have

Corollary 3.5 Define the sequences $z=\left(z_{n}\right)$ and $b^{(k)}(\widetilde{r}, \widetilde{s})=\left\{b_{n}^{(k)}(\widetilde{r}, \widetilde{s})\right\}_{n \in \mathbb{N}}$ for every fixed $k \in \mathbb{N}$ by

$$
z_{n}:=\sum_{k=0}^{n} \frac{1}{r_{k}} \prod_{i=0}^{k-1} \frac{-s_{i}}{r_{i}} \quad \text { and } \quad b_{n}^{(k)}(\widetilde{r}, \widetilde{s}):= \begin{cases}0 & (n<k) \\ \frac{1}{r_{n}} \prod_{i=k}^{n-1} \frac{-s_{i}}{r_{i}} & (n \geq k) .\end{cases}
$$

Then

(a) the sequence $\left\{b^{(k)}(\widetilde{r}, \widetilde{s})\right\}_{k \in \mathbb{N}}$ is a basis for the spaces $\widetilde{c}_{0}$ and $\tilde{\ell}_{p}$, and any $x$ in $\widetilde{c}_{0}$ or in $\tilde{\ell}_{p}$ has a unique representation of the form

$$
x:=\sum_{k} \alpha_{k}(r) b^{(k)}(\widetilde{r}, \widetilde{s})
$$

where $\alpha_{k}(r):=\{B(\tilde{r}, \tilde{s}) x\}_{k}$ for all $k \in \mathbb{N}$.

(b) the set $\left\{z, b^{(k)}(\tilde{r}, \widetilde{s})\right\}$ is a basis for the space $\widetilde{c}$, and any $x$ in $\widetilde{c}$ has a unique

representation of the form

$$
x:=l z+\sum_{k}\left[\alpha_{k}(r)-l\right] b^{(k)}(\tilde{r}, \widetilde{s})
$$

where $l:=\lim _{k \rightarrow \infty}\{B(\widetilde{r}, \widetilde{s}) x\}_{k}$.

By $\lambda \mu$, we mean the set

$$
\lambda \mu:=\left\{z=\left(z_{k}\right) \in \omega: z_{k}=x_{k} y_{k} \forall k \in \mathbb{N}, x=\left(x_{k}\right) \in \lambda, y=\left(y_{k}\right) \in \mu\right\}
$$

for the sequence spaces $\lambda$ and $\mu$.

Given a $B K$-space $\lambda \supset \phi$, we denote the $n$th section of a sequence $x=\left(x_{k}\right) \in \lambda$ by $x^{[n]}:=$ $\sum_{k=0}^{n} x_{k} e^{(k)}$, and we say that $x$ has the property

$A K$ if $\lim _{n \rightarrow \infty}\left\|x-x^{[n]}\right\|_{\lambda}=0$ (abschnittskonvergenz),

$A B$ if $\sup _{n \in \mathbb{N}}\left\|x^{[n]}\right\|_{\lambda}<\infty$ (abschnittsbeschränktheit),

$A D$ if $x \in \bar{\phi}$ (closure of $\phi \subset \lambda$ ) (abschnittsdichte),

$K B$ if the set $\left\{x_{k} e^{(k)}\right\}$ is bounded in $\lambda$ (koordinatenweise beschränkt). 
If one of these properties holds for every $x \in \lambda$, then we say that the space $\lambda$ has that property (cf. [61]). It is trivial that $A K$ implies $A D$ and $A K$ iff $A B$ and $A D$. For example, $c_{0}$ and $\ell_{p}$ are $A K$-spaces and $c$ and $\ell_{\infty}$ are not $A D$-spaces.

The sequence space $\lambda$ is said to be solid if and only if

$$
\tilde{\lambda}:=\left\{\left(u_{k}\right) \in \omega: \exists\left(x_{k}\right) \in \lambda \text { such that }\left|u_{k}\right| \leq\left|x_{k}\right| \text { for all } k \in \mathbb{N}\right\} \subset \lambda \text {. }
$$

For a sequence $J$ of $\mathbb{N}$ and a sequence space $\lambda$, we define $\lambda_{J}$ by

$$
\lambda_{J}:=\left\{x=\left(x_{i}\right): \text { there is a } y=\left(y_{i}\right) \in \lambda \text { with } x_{i}=y_{n_{i}}, \forall n_{i} \in J\right\}
$$

and call $\lambda_{J}$ the $J$-stepspace or a $J$-sectional subspace of $\lambda$. If $x_{J} \in \lambda_{J}$, then the canonical preimage of $x_{J}$ is the sequence $\bar{x}_{J}$ which agrees with $x_{J}$ on the indices in $J$ and is zero elsewhere. Then $\lambda$ is called monotone provided $\lambda$ contains the canonical preimages of all its stepspaces.

Lemma 3.6 [46, Theorem 2.1 and Lemma 4.1] Let $\lambda, \mu$ be the BK-spaces and $C_{\mu}^{U}=\left(c_{n k}\right)$ be defined via the sequence $\alpha=\left(\alpha_{k}\right) \in \mu$ and the triangle matrix $U=\left(u_{n k}\right)$ by

$$
c_{n k}:=\sum_{j=k}^{n} \alpha_{j} u_{n j} v_{j k}
$$

for all $k, n \in \mathbb{N}$. Then the domain of the matrix $U$ in the sequence space $\lambda$ has the property

(i) $K B$ if and only if $C_{\ell_{1}}^{U} \in(\lambda: \lambda)$,

(ii) $A B$ if and only if $C_{b v_{0}}^{U} \in(\lambda: \lambda)$,

(iii) monotone if and only if $C_{m_{0}}^{U} \in(\lambda: \lambda)$,

(iv) solid if and only if $C_{\ell_{\infty}}^{U} \in(\lambda: \lambda)$.

From Lemma 3.6, we have

Corollary 3.7 If $s_{n}=r_{n}$ for all $n \in \mathbb{N}$, then $\tilde{\ell}_{1}$ has the $K B$ - and AB-properties.

Lemma 3.8 [46, Theorem 2.2] Let $\lambda$ be a BK-space which has the AK-property, $U$ be a triangle matrix and $\lambda_{U} \supset \phi$. Then the sequence space $\lambda_{U}$ has the AD-property if and only if the fact $t U=\theta$ for $t \in \lambda^{\beta}$ implies the fact $t=\theta$.

Since $c_{0}$ and $\ell_{p}$ have the $A K$-property, we can employ Lemma 3.8 for the matrix $U=$ $B(\widetilde{r}, \widetilde{s})$. Then we have

Corollary $3.9 \widetilde{c}_{0}$ and $\tilde{\ell}_{p}(p>1)$ have the AD-property if and only if $s_{n} \leq r_{n}$ for all $n \in \mathbb{N}$.

4 Some matrix transformations related to the sequence spaces $\tilde{l}_{\infty}, \tilde{c}_{,} \tilde{c}_{0}$ and $\tilde{\ell}_{1}$ In the present section, we characterize some classes of infinite matrices related to new sequence spaces.

Theorem 4.1 Let $\lambda$ be an FK-space, $U$ be a triangle, $V$ be its inverse and $\mu$ be an arbitrary subset of $\omega$. Then we have $A=\left(a_{n k}\right) \in\left(\lambda_{U}: \mu\right)$ if and only if

$$
C^{(n)}=\left(c_{m k}^{(n)}\right) \in(\lambda: c) \quad \text { for all } n \in \mathbb{N}
$$


and

$$
C=\left(c_{n k}\right) \in(\lambda: \mu)
$$

where

$$
c_{m k}^{(n)}:=\left\{\begin{array}{ll}
\sum_{j=k}^{m} a_{n j} v_{j k} & (0 \leq k \leq m), \\
0 & (k>m)
\end{array} \quad \text { and } \quad c_{n k}:=\sum_{j=k}^{\infty} a_{n j} v_{j k} \quad \text { for all } k, m, n \in \mathbb{N} .\right.
$$

Proof Let $A=\left(a_{n k}\right) \in\left(\lambda_{U}: \mu\right)$ and take $x \in \lambda_{U}$. Then we obtain the equality

$$
\begin{aligned}
\sum_{k=0}^{m} a_{n k} x_{k} & =\sum_{k=0}^{m} a_{n k}\left(\sum_{j=0}^{k} v_{k j} y_{j}\right) \\
& =\sum_{k=0}^{m}\left(\sum_{j=k}^{m} a_{n j} v_{j k}\right) y_{k}=\sum_{k=0}^{m} c_{n k}^{(n)} y_{k}
\end{aligned}
$$

for all $m, n \in \mathbb{N}$. Since $A x$ exists, $C^{(n)}$ must belong to the class $(\lambda: c)$. Letting $m \rightarrow \infty$ in equality (4.3) we have $A x=C y$. Since $A x \in \mu$, then $C y \in \mu$, i.e., $C \in(\lambda: \mu)$.

Conversely, let (4.1), (4.2) hold and take $x \in \lambda_{U}$. Then we have $\left(c_{n k}\right)_{k \in \mathbb{N}} \in \lambda^{\beta}$, which together with (4.1) gives that $\left(a_{n k}\right)_{k \in \mathbb{N}} \in \lambda_{U}^{\beta}$ for all $n \in \mathbb{N}$. Hence, $A x$ exists. Therefore, we obtain from equality (4.3) as $m \rightarrow \infty$ that $A x=C y$ and this shows that $A \in\left(\lambda_{U}: \mu\right)$.

Now, we list the following conditions:

$$
\begin{aligned}
& \sup _{m \in \mathbb{N}} \sum_{k=0}^{m}\left|\sum_{j=k}^{m} \frac{1}{r_{j}} \prod_{i=k}^{j-1} \frac{-s_{i}}{r_{i}} a_{n j}\right|^{q}<\infty, \\
& \lim _{m \rightarrow \infty} \sum_{j=k}^{m} \frac{1}{r_{j}} \prod_{i=k}^{j-1} \frac{-s_{i}}{r_{i}} a_{n j}=c_{n k}, \\
& \lim _{m \rightarrow \infty} \sum_{k=0}^{m}\left|\sum_{j=k}^{m} \frac{1}{r_{j}} \prod_{i=k}^{j-1} \frac{-s_{i}}{r_{i}} a_{n j}\right|=\sum_{k}\left|c_{n k}\right| \quad \text { for each } n \in \mathbb{N}, \\
& \lim _{m \rightarrow \infty} \sum_{k=0}^{m} \sum_{j=k}^{m} \frac{1}{r_{j}} \prod_{i=k}^{j-1} \frac{-s_{i}}{r_{i}} a_{n j}=\alpha_{n} \quad \text { for each } n \in \mathbb{N}, \\
& \sup _{k, m \in \mathbb{N}}\left|\sum_{j=k}^{m} \frac{1}{r_{j}} \prod_{i=k}^{j-1} \frac{-s_{i}}{r_{i}} a_{n j}\right|<\infty, \\
& \sup _{n \in \mathbb{N}} \sum_{k}\left|c_{n k}\right|^{q}<\infty, \\
& \lim _{n \rightarrow \infty} c_{n k}=\beta_{k}, \\
& \lim _{n \rightarrow \infty} \sum_{k}\left|c_{n k}\right|=\sum_{n \rightarrow \infty} \sum_{k}\left|\beta_{k}\right|, \\
& \lim _{n k}=\beta,
\end{aligned}
$$


Table 3 The characterization of the class $(\tilde{\lambda}: \mu)$ with $\lambda \in\left\{\ell_{\infty}, c, c_{0}, \ell_{p}, \ell_{1}\right\}$ and $\mu \in\left\{\ell_{\infty}, c, c_{0}, \ell_{1}\right\}$

\begin{tabular}{lrrrrr}
\hline To & \multicolumn{3}{l}{ From } & & \\
\cline { 2 - 6 } & $\tilde{\boldsymbol{\ell}}_{\boldsymbol{\infty}}$ & $\tilde{\boldsymbol{c}}$ & $\tilde{\boldsymbol{c}}_{\mathbf{0}}$ & $\tilde{\boldsymbol{\ell}}_{\boldsymbol{p}}$ & $\tilde{\boldsymbol{\ell}}_{\mathbf{1}}$ \\
\hline$\ell_{\infty}$ & 1. & 2. & 3. & 4. & 5. \\
$c$ & 6. & 7. & 8. & 9. & 10. \\
$c_{0}$ & 11. & 12. & 13. & 14. & 15. \\
$\boldsymbol{\ell}_{1}$ & 16. & 17. & 18. & 19. & 20. \\
\hline
\end{tabular}

Here 1. (4.5), (4.6) and (4.9) with $q=1.2$. (4.5), (4.7) and (4.4), (4.9) with $q=1$. 3. (4.5) and (4.4), (4.9) with $q=1.4$. (4.4), (4.5) and (4.9). 5. (4.5), (4.8) and (4.13). 6. (4.5), (4.6), (4.10) and (4.11). 7. (4.5), (4.7), (4.10), (4.12) and (4.4), (4.9) with $q=1.8$. (4.5), (4.10) and (4.4), (4.9) with $q=1.9 .(4.4),(4.5),(4.9)$ and (4.10). 10. (4.5), (4.8), (4.10) and (4.13). 11. (4.5), (4.6) and (4.15). 12. (4.5), (4.7), (4.10) with $\beta_{k}=0$ and (4.12) with $\beta=0$, and (4.4), (4.9) with $q=1$. 13. (4.5), (4.10) with $\beta_{k}=0$ and (4.4), (4.9) with $q=1$. 14. (4.4), (4.5), (4.9) and (4.10) with $\beta_{k}=0.15$. (4.5), (4.8), (4.10) with $\beta_{k}=0$ and (4.13). 16. (4.5), (4.6) and (4.16). 17. (4.4) with $q=1,(4.5)$, (4.7) and (4.16). 18. (4.4) with $q=1,(4.5)$ and (4.16). 19. (4.4), (4.5) and (4.17). 20. (4.5), (4.8) and (4.14).

$$
\begin{aligned}
& \sup _{k, n \in \mathbb{N}}\left|c_{n k}\right|<\infty, \\
& \sup _{k \in \mathbb{N}} \sum_{n}\left|c_{n k}\right|<\infty, \\
& \lim _{n \rightarrow \infty} \sum_{k} c_{n k}=0, \\
& \sup _{N, K \in \mathcal{F}}\left|\sum_{n \in N} \sum_{k \in K} c_{n k}\right|<\infty, \\
& \sup _{N \in \mathcal{F}} \sum_{k}\left|\sum_{n \in N} c_{n k}\right|^{q}<\infty,
\end{aligned}
$$

where $\mathcal{F}$ denotes the collection of all finite subsets of $\mathbb{N}$.

We have from Theorem 4.1

Corollary 4.2 The necessary and sufficient conditions for $A \in(\lambda: \mu)$ when $\lambda \in\left\{\widetilde{\ell}_{\infty}, \widetilde{c}, \widetilde{c}_{0}\right.$, $\left.\tilde{\ell}_{p}, \tilde{\ell}_{1}\right\}$ and $\mu \in\left\{\ell_{\infty}, c, c_{0}, \ell_{1}\right\}$ can be read from Table 3 .

Now, we may present our final lemma given by Başar and Altay [18, Lemma 5.3] which is useful for obtaining the characterization of some new matrix classes from Corollary 4.2.

Lemma 4.3 Let $\lambda, \mu$ be any two sequence spaces, $A$ be an infinite matrix and $U$ be a triangle matrix. Then $A \in\left(\lambda: \mu_{U}\right)$ if and only if $U A \in(\lambda: \mu)$.

We should finally note that if $a_{n k}$ is replaced by $r_{n} a_{n k}+s_{n-1} a_{n-1, k}$ for all $k, n \in \mathbb{N}$ in Corollary 4.2 , then one can derive the characterization of the class $(\tilde{\lambda}: \tilde{\mu})$ from Lemma 4.3 with $U=B(\widetilde{r}, \widetilde{s})$.

\section{Conclusion}

Quite recently, Kirişçi and Başar [44] studied the domain of the generalized difference matrix $B(r, s)$ in the classical sequence spaces $\ell_{\infty}, c, c_{0}$ and $\ell_{p}$. Later, Sönmez [45] generalized these results by using the triple band matrix $B(r, s, t)$. Since the generalized difference matrix $B(r, s)$ is obtained in the special case $r_{n}=r$ and $s_{n}=s$ for all $n \in \mathbb{N}$ from the double sequential band matrix $B(\widetilde{r}, \widetilde{s})$, our results are much more general than the corresponding results given by Kirişçi and Başar [44]. 
Finally, we should note that our next paper will be devoted to the investigation of the domain of the double sequential band matrix $B(\widetilde{r}, \widetilde{s})$ in the space $f$ of almost convergent sequences introduced by Lorentz in [62] which generalizes the corresponding results of Başar and Kirişçi [63].

\section{Competing interests}

The author declares that they have no competing interests.

\section{Acknowledgements}

The author would like to express their gratitude to professor Bilal Altay, Department of Mathematical Education, İnönü University, 44280 Malatya-Turkey, for making some constructive comments on the main results of the earlier version of the manuscript which improved the presentation of the paper.

Received: 4 January 2012 Accepted: 19 November 2012 Published: 5 December 2012

\section{References}

1. Wang, C-S: On Nörlund sequence spaces. Tamkang J. Math. 9, 269-274 (1978)

2. Ng, P-N, Lee, P-Y: Cesàro sequence spaces of non-absolute type. Ann. Soc. Math. Pol., 1 Comment. Math. 20(2), 429-433 (1978)

3. Et, M: On some generalized Cesàro difference sequence spaces. Istanb. Üniv. Fen Fak. Mat. Derg. 55-56, 221-229 (1996-1997)

4. Malkowsky, E: Recent results in the theory of matrix transformations in the sequence spaces. Mat. Vesn. 49, 187-196 (1997)

5. Kızmaz, H: On certain sequence spaces. Can. Math. Bull. 24(2), 169-176 (1981)

6. Et, M: On some difference sequence spaces. Turk. J. Math. 17, 18-24 (1993)

7. Mursaleen, M: Generalized spaces of difference sequences. J. Math. Anal. Appl. 203(3), 738-745 (1996)

8. Malkowsky, E, Savaş, E: Matrix transformations between sequence spaces of generalized weighted means. Appl. Math. Comput. 147, 333-345 (2004)

9. Şengönül, M, Başar, F: Some new Cesàro sequence spaces of non-absolute type which include the spaces $C_{0}$ and $c$. Soochow J. Math. 31(1), 107-119(2005)

10. Altay, B, Başar, F: Some Euler sequence spaces of non-absolute type. Ukr. Math. J. 57(1), 1-17 (2005)

11. Rhoades, BE: Some sequence spaces which include $c_{0}$ and C. Hokkaido Math. J. 35, 587-599 (2006)

12. Aydın, C, Başar, F: On the new sequence spaces which include the spaces $C_{0}$ and c. Hokkaido Math. J. 33(2), 383-398 (2004)

13. Aydın, C, Başar, F: Some new sequence spaces which include the spaces $\ell_{p}$ and $\ell_{\infty}$. Demonstr. Math. 38(3), 641-656 (2005)

14. Altay, B, Başar, F, Mursaleen, M: On the Euler sequence spaces which include the spaces $\ell_{p}$ and $\ell_{\infty}$ I. Inf. Sci. 176(10), 1450-1462 (2006)

15. Mursaleen, $M$, Başar, F, Altay, B: On the Euler sequence spaces which include the spaces $\ell_{p}$ and $\ell_{\infty}$ II. Nonlinear Anal. TMA 65(3), 707-717 (2006)

16. Aydın, C, Başar, F: Some new difference sequence spaces. Appl. Math. Comput. 157(3), 677-693 (2004)

17. Khan, FM, Rahman, MF: Matrix transformations on Cesà ro sequence spaces of nonabsolute type. J. Anal. 4, 97-101 (1996)

18. Başar, F, Altay, B: On the space of sequences of p-bounded variation and related matrix mappings. Ukr. Math. J. 55(1), 136-147 (2003)

19. Çolak, R, Et, M, Malkowsky, E: Some Topics of Sequence Spaces. Lecture Notes in Mathematics. Fırat Univ. Press, Elazığ (2004). ISBN:975-394-0386-6

20. Altay, B, Başar, F: The matrix domain and the fine spectrum of the difference operator $\Delta$ on the sequence space $\ell_{p}$ $(0<p<1)$. Commun. Math. Anal. 2(2), 1-11 (2007)

21. Et, M, Çolak, R: On some generalized difference sequence spaces. Soochow J. Math. 21(4), 377-386 (1995)

22. Çolak, R, Et, M: On some generalized sequence spaces and related matrix transformations. Hokkaido Math. J. 26(3), 483-492 (1997)

23. Altay, B: On the space of $p$-summable difference sequences of order $m,(1 \leq p<\infty)$. Studia Sci. Math. Hung. 43(4), 387-402 (2006)

24. Malkowsky, E, Parashar, SD: Matrix transformations in space of bounded and convergent difference sequence of order m. Analysis 17, 87-97 (1997)

25. Polat, H, Başar, F: Some Euler spaces of difference sequences of order m. Acta Math. Sci. 27(2), 254-266 (2007)

26. Altay, B, Başar, F, Malkowsky, E: Matrix transformations on some sequence spaces related to strong Cesàro summability and boundedness. Appl. Math. Comput. 211, 255-264 (2009)

27. Başar, F, Malkowsky, E, Altay, B: Matrix transformations on the matrix domains of triangles in the spaces of strongly $C_{1}$-summable and bounded sequences. Publ. Math. 73(1-2), 193-213 (2008)

28. Başar, F: Infinite matrices and almost boundedness. Boll. Unione Mat. Ital., A 6(3), 395-402 (1992)

29. Başar, F, Altay, B: Matrix mappings on the space $b s(p)$ and its $\alpha$-, $\beta$ - and $\gamma$-duals. Aligarh Bull. Math. 21(1), 79-91 (2001)

30. Aydın, C, Başar, F: Some generalizations of the sequence space $a_{p}^{r}$. Iran. J. Sci. Technol., Trans. A, Sci. 30(A2), 175-190 (2006)

31. Aydın, C, Başar, F: Some topological and geometric properties of the domain of the generalized difference matrix $B(r, s)$ in the sequence spaces I(p). Serbia, June 2009, Book of Abstracts p.p. 1 
32. Choudhary, B, Mishra, SK: On Köthe-Toeplitz duals of certain sequence spaces and their matrix transformations. Indian J. Pure Appl. Math. 24(5), 291-301 (1993)

33. Ahmad, ZU, Mursaleen, M: Köthe-Toeplitz duals of some new sequence spaces and their matrix maps. Publ. Inst. Math. (Belgr.) 42, 57-61 (1987)

34. Asma, Ç, Çolak, R: On the Köthe-Toeplitz duals of some generalized sets of difference sequences. Demonstr. Math. 33, 797-803 (2000)

35. Bektaş, ÇA: On some new generalized sequence spaces. J. Math. Anal. Appl. 277, 681-688 (2003)

36. Altay, B, Başar, F: Some paranormed sequence spaces of non-absolute type derived by weighted mean. J. Math. Anal. Appl. 319(2), 494-508 (2006)

37. Altay, B, Başar, F: Generalization of the sequence space $\ell(p)$ derived by weighted mean. J. Math. Anal. Appl. 330(1), 174-185 (2007)

38. Başar, F, Altay, B, Mursaleen, M: Some generalizations of the space $b v_{p}$ of $p$-bounded variation sequences. Nonlinear Anal. 68(2), 273-287 (2008)

39. Aydın, C, Başar, F: Some new paranormed sequence spaces. Inf. Sci. 160(1-4), 27-40 (2004)

40. Altay, B, Başar, F: On the paranormed Riesz sequence spaces of non-absolute type. Southeast Asian Bull. Math. 26(5), 701-715 (2002)

41. Altay, B, Başar, F: Some paranormed Riezs sequence spaces of non-absolute type. Southeast Asian Bull. Math. 30(5), 591-608 (2006)

42. Et, M, Başarır, M: On some new generalized difference sequence spaces. Period. Math.-Phys 10, 63-71 (1987)

43. Malkowsky, E, Mursaleen, M, Suantai, S: The dual spaces of sets of difference sequences of order $m$ and matrix transformations. Acta Math. Sin. Engl. Ser. 23(3), 521-532 (2007)

44. Kiriş̧̧i, M, Başar, F: Some new sequence spaces derived by the domain of generalized difference matrix. Comput. Math. Appl. 60(5), 1299-1309 (2010)

45. Sönmez, A: Some new sequence spaces derived by the domain of the triple band matrix. Comput. Math. Appl. 62(2), 641-650 (2011)

46. Altay, B, Basar, F: Certain topological properties and duals of the matrix domain of a triangle matrix in a sequence space. J. Math. Anal. Appl. 336(1), 632-645 (2007)

47. Başarı, M: On some new sequence spaces and related matrix transformations. Indian J. Pure Appl. Math. 26(10), 1003-1010 (1995)

48. Sarıgöl, MA: On difference sequence spaces. J. Karadeniz Tech. Univ. Fac. Arts Sci. Ser. Math.-Phys. 10, 63-71 (1987)

49. Malkowsky, E: Absolute and ordinary Köthe-Toeplitz duals of some sets of sequences and matrix transformations. Publ. Inst. Math. (Beograd) (N.S.) 46(60), 97-103 (1989)

50. Choudhary, B, Mishra, SK: A note on certain sequence spaces. J. Anal. 1, 139-148 (1993)

51. Mishra, SK: Matrix maps involving certain sequence spaces. Indian J. Pure Appl. Math. 24(2), 125-132 (1993)

52. Mursaleen, M, Gaur, AK, Saif, AH: Some new sequence spaces and their duals and matrix transformations. Bull. Calcutta Math. Soc. 88(3), 207-212 (1996)

53. Gnanaseelan, C, Srivastava, PD: The $\alpha$-, $\beta$ - and $\gamma$-duals of some generalised difference sequence spaces. Indian J. Math. 38(2), 111-120 (1996)

54. Malkowsky, E: A note on the Köthe-Toeplitz duals of generalized sets of bounded and convergent difference sequences. J. Anal. 4, 81-91 (1996)

55. Gaur, AK, Mursaleen, M: Difference sequence spaces. Int. J. Math. Math. Sci. 21(4), 701-706 (1998)

56. Malkowsky, E, Mursaleen, M, Qamaruddin: Generalized sets of difference sequences, their duals and matrix transformations. In: Sequence Spaces and Applications, pp. 68-83. Narosa, New Delhi (1999)

57. Malkowsky, E, Mursaleen, M: Some matrix transformations between the difference sequence spaces $\Delta c_{0}(p), \Delta c(p)$ and $\Delta \ell_{\infty}(p)$. Filomat $15,353-363(2001)$

58. Kamthan, PK, Gupta, M: Sequence Spaces and Series. Dekker, New York (1981)

59. Stieglitz, M, Tietz, H: Matrix transformationen von folgenräumen eine ergebnisübersicht. Math. Z. 154, 1-16 (1997)

60. Jarrah, AM, Malkowsky, E: BK spaces, bases and linear operators. Rend. Circ. Mat. Palermo (2) 52, 177-191 (1990)

61. Grosse-Erdmann, K-G: On I'-invariant sequence spaces. J. Math. Anal. Appl. 262, 112-132 (2001)

62. Lorentz, GG: A contribution to the theory of divergent sequences. Acta Math. 80, 167-190 (1948)

63. Başar, F, Kiriş̧̧i, M: Almost convergence and generalized difference matrix. Comput. Math. Appl. 61(3), 602-611 (2011)

doi:10.1186/1029-242X-2012-281

Cite this article as: Candan: Domain of the double sequential band matrix in the classical sequence spaces. Journal of Inequalities and Applications 2012 2012:281. 